\title{
A Neural Circuit That Controls Cortical State, Plasticity, and the Gain of Sensory Responses in Mouse
}

\author{
Michael P. StryKer \\ Center for Integrative Neuroscience, Department of Physiology, University of California, \\ San Francisco, California 94143-0444 \\ Correspondence: stryker@phy.ucsf.edu
}

\begin{abstract}
Neurons in the visual cortex were first found to be exquisitely selective for particular properties of visual stimuli in anesthetized animals, including mice. Studies of alert mice in an apparatus that allowed them to stand or run revealed that locomotion causes a change in cortical state that dramatically increases the magnitude of responses in neurons of the visual cortex without altering selectivity, effectively changing the gain of sensory responses. Locomotion also dramatically enhances adult plasticity in the recovery from long-term visual deprivation. We have studied the elements and operation of the neural circuit responsible for the enhancement of activity and shown that it enhances plasticity even in mice not free to run. The circuit consists of projections ascending from the midbrain locomotor region (MLR) to the basal forebrain, activating cholinergic and perhaps other projections to excite inhibitory interneurons expressing vasoactive intestinal peptide (VIP) in the visual cortex. VIP cells activated by locomotion inhibit interneurons that express somatostatin (SST), thereby disinhibiting the excitatory principal neurons and allowing them to respond more strongly to effective visual stimuli. These findings reveal in alert animals how the ascending reticular activating system described in anesthetized animals 50 years ago operates to control cortical state.
\end{abstract}

Excitatory neurons in the upper layers of the primary visual cortex (V1) of the mouse, like those in the cat and monkey that were studied 50 years ago (Hubel and Wiesel 1962), are highly selective for particular visual stimuli (Niell and Stryker 2008). Most are classic simple cells, with extended regions in the visual field responding to light increment and others responding to light decrement and generating responses at a limited range of orientations of a bar or edge stimulus and over a limited range of spatial frequencies of a grating. The preferred orientation and spatial frequency can be predicted from the arrangement of the $\mathrm{ON}$ and OFF regions as mapped with flashing spots (Figs. 1 and 2).

\section{LOCOMOTION INCREASES THE GAIN OF VISUAL RESPONSES}

Studying receptive fields of alert mice whose heads were fixed in an apparatus that allowed them to stand or run freely on a large Styrofoam ball floating on air (Dombeck et al. 2007) revealed a new phenomenon: Neural responses to preferred visual stimuli became dramatically larger when the mice started to walk or run but were no less selective than when the mice were stationary or anesthetized (Niell and Stryker 2010). Locomotion appeared simply to multiply the tuning functions of response as a function of orientation or spatial frequency by a constant factor for each neuron, like changing the gain of an amplifier. No similar changes were evident in simultaneous recordings from the lateral geniculate nucleus, the source of visual input to V1, revealing that the gain changes were central, taking place in the cortex and not in the eyes or the thalamus (Fig. 3).

\section{LOCOMOTION ENHANCES ADULT PLASTICITY}

In the mouse, as in the human and the carnivores and primates studied earlier, visual responses to the two eyes can be dramatically and lastingly perturbed by altered visual experience during a brief critical period in early life (Fig. 4) (Gordon and Stryker 1996). Blurring or occluding the vision of one eye during this critical period of susceptibility to the effects of monocular visual deprivation leads to a permanent reduction of visual acuity and impairment of other visual functions, a condition referred to as amblyopia ex anopsia. This form of amblyopia is associated with a pruning of the terminal arbors of thalamocortical afferents to V1 serving the deprived eye. In the cat, this results in a loss of $50 \%$ of the synaptic boutons and arbor length after a week of deprivation (Antonini and Stryker 1993). Not surprisingly, given the loss of much of the deprived-eye input to V1, longstanding amblyopia induced during the critical period is generally refractory to therapy in humans and in carnivores and primates. In contrast to the dramatic plasticity observed during the critical period, monocular visual deprivation in adulthood, or indeed at any time after the critical period, has little lasting effect in these species. Although visual deprivation does produce changes in visual responses in adult mice, the adult plasticity is significantly slower, smaller, and qualitatively different from critical period plasticity (Sato and Stryker 2008). 
A
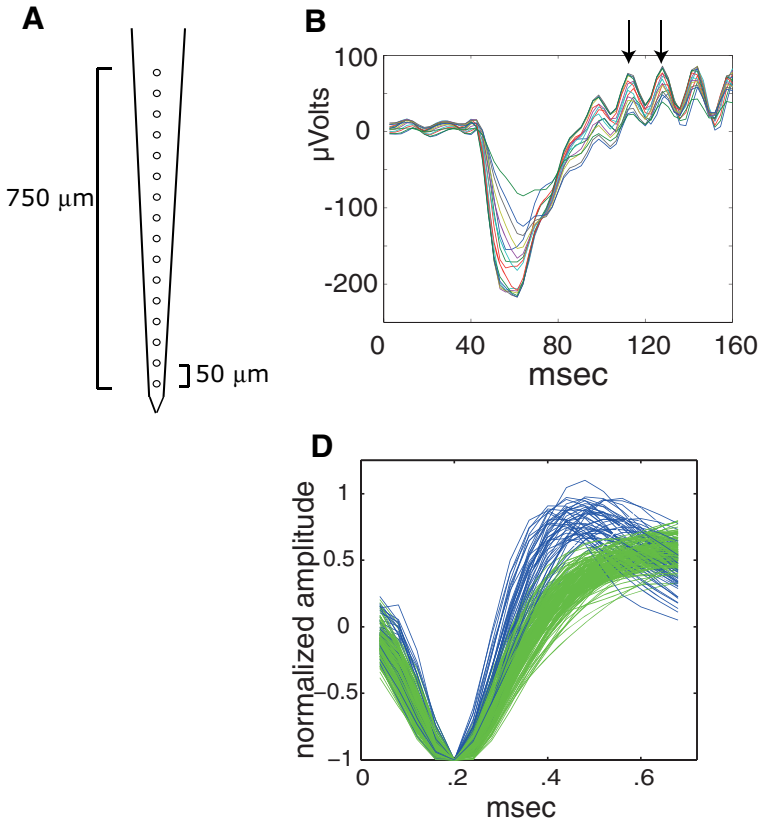

C

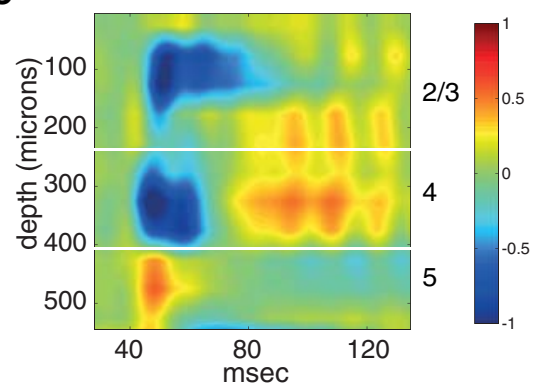

E

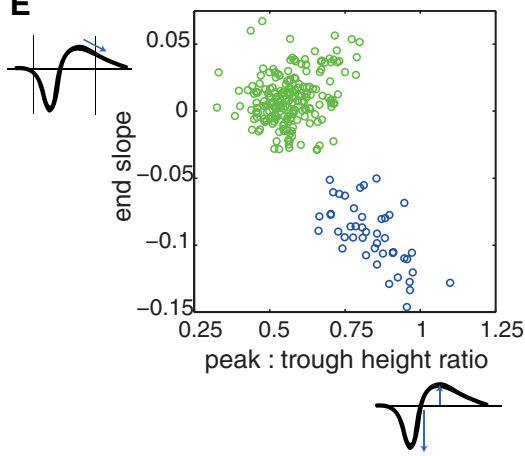

Figure 1. Multisite recording and analysis technology that made characterization of mouse visual cortex feasible by studying an average of more than 10 neurons simultaneously, determining cortical layer containing each recording site, and classifying spike waveforms as presumptive excitatory or inhibitory with no overlap. $(A)$ Schematic of linear multisite probe. $(B)$ Average local field potential (LFP) responses for 16 sites through the depth of cortex. Arrows show consecutive peaks of the high-frequency oscillation. $(C)$ Current source density (CSD) analysis of traces in $B$ reveals positions of cortical layers. $(D)$ Average spike waveforms for all units analyzed $(n=231)$, normalized by trough depth, demonstrating narrow-spiking (blue), and broad-spiking (green) units. (E) Scatter plot of spike waveform parameters for all units. (Data from Niell and Stryker 2008.)

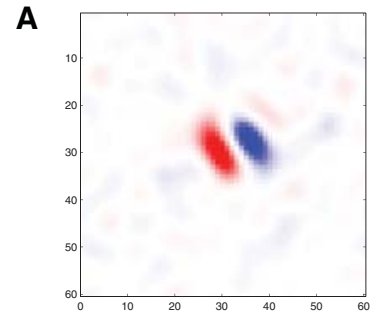

$\mathbf{E}$

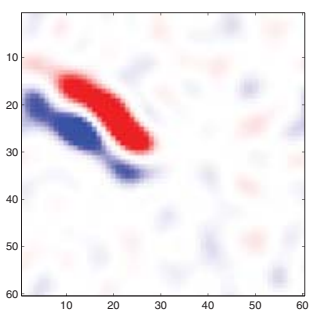

B

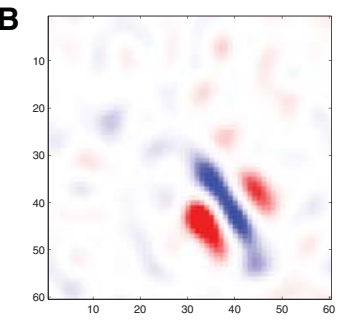

$\mathbf{F}$

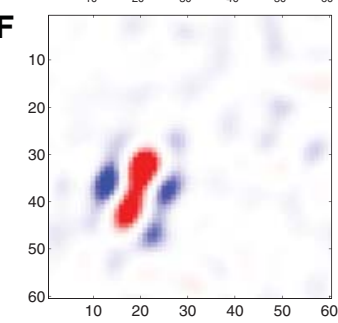

C

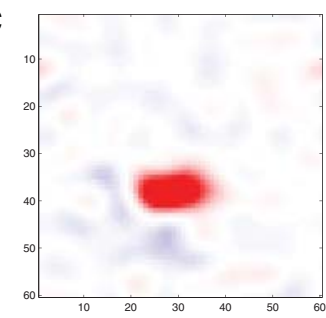

G

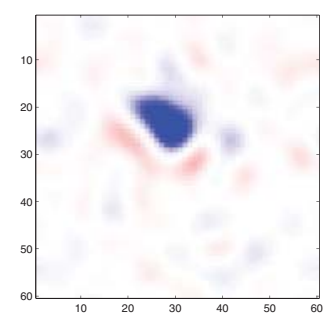

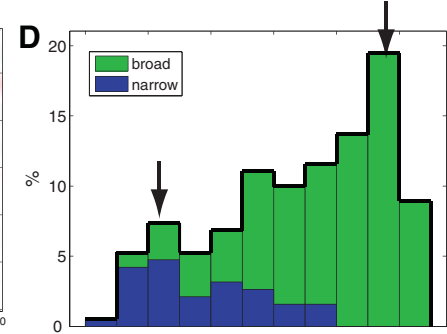

H

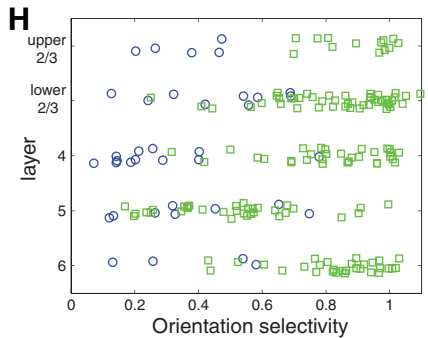

Figure 2. Receptive fields in normal mouse visual cortex measured by spike-triggered averaging $(A-C, E-H)$. Examples of spatial receptive fields with two, three, and one subfield, respectively, showing varying orientation, ON/OFF centers, and spacing of subfields. Red indicates ON responses; blue indicates OFF responses. (D) Distribution of orientation selectivity in broad-spiking (presumptive excitatory) and narrow-spiking (presumptive inhibitory) cells. $(H)$ Orientation selectivity of individual neurons in relation to cortical layer. Note that nearly all broad-spiking cells in the upper layers are highly selective. $(A-D, F$, Reprinted from Niell and Stryker 2008, with permission from the Society for Neuroscience.) 

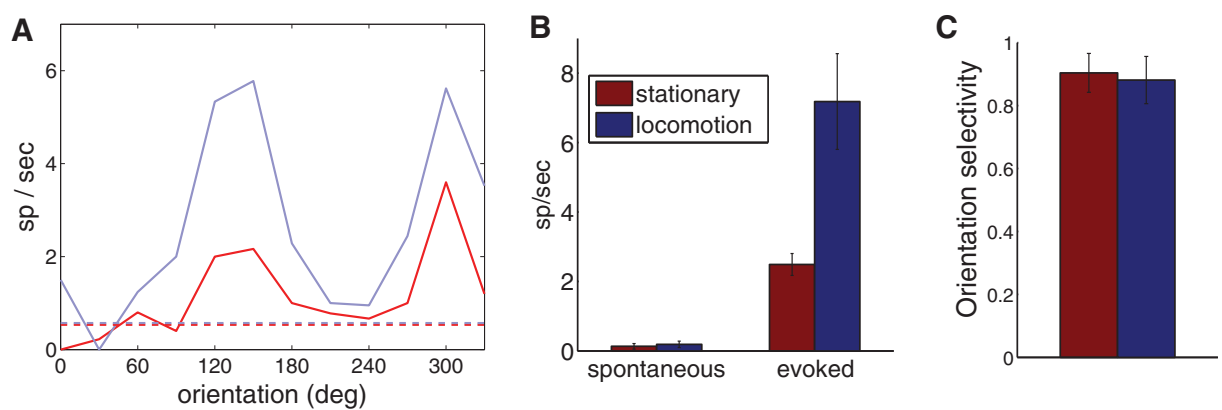

Figure 3. Enhancement of visual responses by locomotion in primary visual cortex of alert mice. $(A)$ Visual response to a drifting grating as a function of direction of movement during locomotion (blue) and while stationary (red). (B) Average spontaneous and visually evoked responses in all broad-spiking neurons in layers 2-4. (C) Average selectivity for stimulus orientation in all broadspiking neurons in layers $2-4$. Note that locomotion increases magnitude of response without changing spontaneous activity or stimulus selectivity. (Data from Niell and Stryker 2010.)

In mice and rats, a number of manipulations were reported to enhance plasticity in adult animals after the end of critical period. Some manipulations produced rapid changes in balance of $\mathrm{V} 1$ responses to the two eyes as a result of monocular deprivation during adulthood. Others were reported to facilitate partial or complete recovery in adults from the effects of longstanding amblyopia induced during the critical period. The effective manipulations included housing or rearing in an enriched environment (containing toys, other mice, and space to climb and jump and run) and administration of antidepressants or other drugs (Sale et al. 2007; Maya Veten- court et al. 2008; Greifzu et al. 2014). It seemed to us plausible that a common feature of these manipulations might be the increased locomotor activity of the subjects. We speculated that the larger visual responses during locomotion would, under any theory of activity-dependent plasticity, give rise to greater plasticity.

To test this hypothesis, we studied the effect of locomotion on recovery from amblyopia in the mouse (Kaneko and Stryker 2014). Imaging the intrinsic optical signal of visual responses through the intact skull is a noninvasive means for precise, quantitative measurement of neural response to the left and right eyes that can be
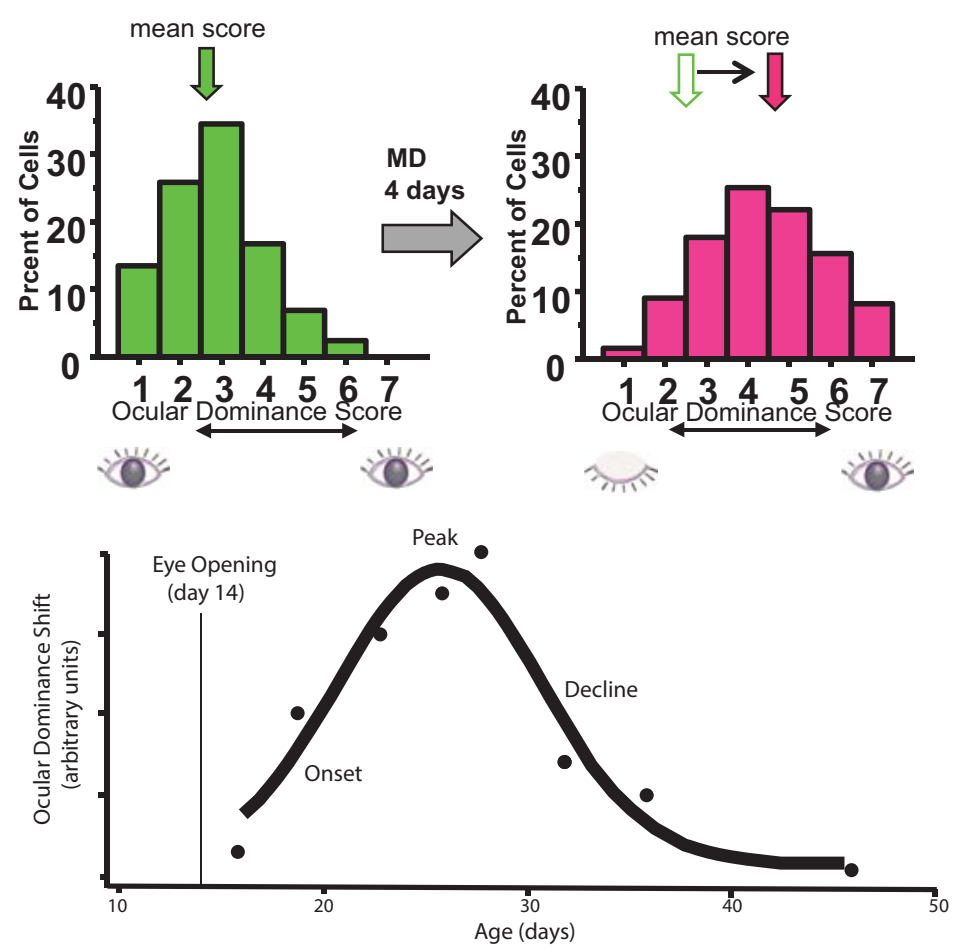

Figure 4. Ocular dominance plasticity during the critical period in mouse visual cortex. (Top) Ocular dominance histograms showing numbers of single neurons as a function of their relative responses to the two eyes before (left, green) and after (right, red) $4 \mathrm{~d}$ of occlusion of vision in the contralateral eye. (Bottom) Time course of the critical period of sensitivity to the effects of monocular visual deprivation (MD) in the primary visual cortex of the mouse. (Adapted from Gordon and Stryker 1996, with permission from the Society for Neuroscience.) 
performed repeatedly on individual animals, making it ideal for tracking recovery of function after deprivation (Kalatsky and Stryker 2003; Kaneko et al. 2008). Mice were deprived of vision in one eye by lid suture through the critical period and continuing into the fifth month of life, leaving them amblyopic. The deprived eye was then opened and the restoration of visual responses was measured. As expected, and as is the case with human amblyopia, responses never recovered more than half way to normal levels in control mice housed in their conventional home cages for the next $3 \mathrm{wk}$. Experimental mice were allowed to run on the Styrofoam ball for $4 \mathrm{~h} / \mathrm{d}$ while viewing high-contrast visual stimuli on a monitor in front of them. These animals rapidly recovered normal levels of intrinsic signal response to the stimuli to which they were exposed. Two additional control groups were studied to determine whether recovery would be produced by locomotion alone, without high-contrast visual stimulation, or by high-contrast visual stimulation alone, without the opportunity for locomotion. Neither of these additional control groups recovered significantly more than those housed in their home cages without visual stimulation. These findings were consistent with our hypothesis that high-gain visual responses might be the common explanation for many phenomena of adult plasticity (Fig. 5).

The recovery from deprivation stimulated by locomotion did not, however, produce a normal visual cortex. Instead, the recovery was highly specific to the stimuli that the animal had viewed during locomotion. Responses to other stimuli that are equally effective in intact mice failed to recover. These findings suggested that plasticity

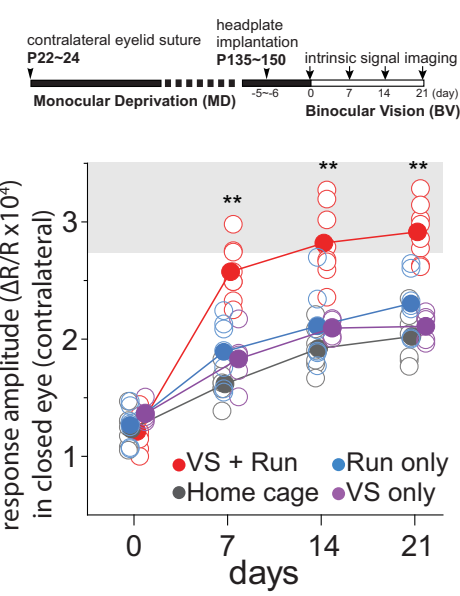

Figure 5. High-contrast visual stimulation during locomotion enhances recovery of visual responses from long-term monocular deprivation. (Top) Experimental timeline. (Bottom) Amplitude of response to contralateral eye, measured by intrinsic signal imaging, as a functions of time after reopening following $\sim 5$ mo of monocular visual deprivation beginning during the critical period. Gray bar indicates the range of response amplitudes in intact mice. Experimental condition (red) with highcontrast visual stimulation during locomotion for $4 \mathrm{~h} / \mathrm{d}$. Control conditions in the home cage (black), with locomotion $4 \mathrm{~h} / \mathrm{d}$ but no high-contrast visual stimulation (blue), or with $8 \mathrm{~h} / \mathrm{d}$ visual stimulation without locomotion in the home cage (magenta). (Adapted from Kaneko and Stryker 2014.) took place only in the specific neural circuits that were activated during the high-gain state.

We wondered how the responses of the vast majority of excitatory neurons in V1 could recover so well, when so much of the excitatory anatomical input to the visual cortex from the lateral geniculate nucleus had been lost (Antonini et al. 1999) and seemed unlikely to have been restored. Recordings before and after recovery from large numbers of presumptive excitatory and inhibitory single neurons, which can be distinguished reliably on the basis of the duration of the waveforms of their action potentials, suggested an answer. Although the visual responses of excitatory neurons showed recovery specific to the stimulus that accompanied locomotion, as described, the firing rates of all the inhibitory neurons were dramatically depressed (Kaneko and Stryker 2014). It appears that the reduction produced by deprivation in thalamocortical excitation is compensated for during recovery by a corresponding reduction in the magnitude of inhibition. The reduced inhibition permits the excitatory-cell responses to increase to normal levels despite reduced excitatory visual input. Such a neural-circuit-dependent homeostatic process may be quite general, supplementing cellular mechanisms for homeostatic regulation of neural activity levels.

\section{THE SUBCORTICAL CIRCUIT ACTIVATED BY LOCOMOTION}

What produces the high-gain state in visual cortex? Because it is turned on by locomotion, exploring the neural circuitry that gives rise to locomotion seemed a promising place to start. Nearly $50 \mathrm{yr}$ ago, research from Orlovskii's laboratory in Moscow revealed a midbrain locomotor region (MLR), within which electrical stimulation induces locomotion at short latencies (Orlovskii et al. 1966). Anatomically, this region loosely coincides with the pedunculopontine tegmental nucleus and the cuneiform nucleus (Garcia-Rill 1991). This area has both descending projections to motor regions and ascending projections, including those to the basal forebrain cholinergic regions that have been shown to modulate behavioral state (Nauta and Kuypers 1958; Skinner et al. 2004). The same region of the brain has been considered to be part of the "ascending reticular activating system," as stimulating this area in anesthetized animals induces physiological changes resembling those of alertness (Fig. 6) (Moruzzi and Magoun 1949).

We injected a virus to transfect excitatory neurons within the MLR with a channelrhodopsin construct, enabling us to activate them optogenetically by shining a blue light onto them through an optical fiber (Lee et al. 2014). Trains of light pulses could reliably make the mice run. We decreased the light pulse intensity until $20 \mathrm{~Hz}$ stimulation was just sufficient to make the mice run at short latency. Not surprisingly, the cortex shifted to the high-gain state during optogenetic simulation, showing visual responses as strong as those elicited during natural running. Most informatively, when the optogenetic stim- 

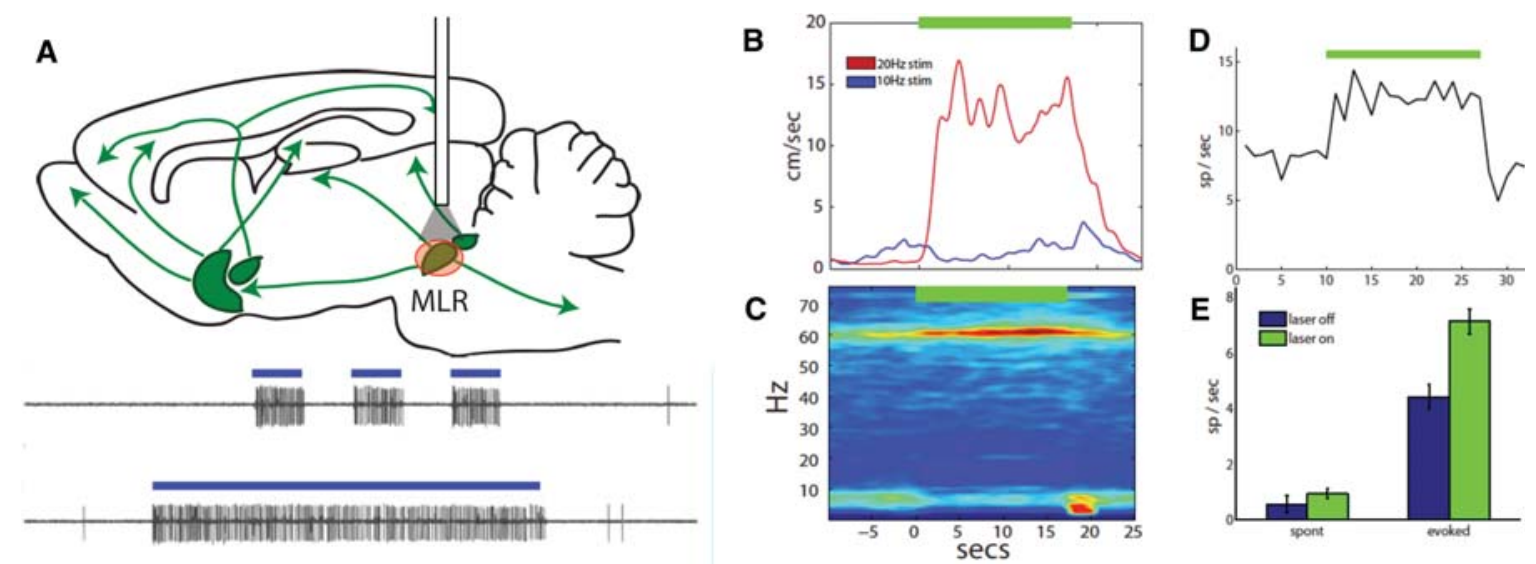

Figure 6. Optogenetic stimulation of excitatory neurons in the midbrain locomotor region (MLR) increases the gain of visual responses in primary visual cortex even without locomotion. (A) Drawing of sagittal section showing MLR, region of channelrhodopsin transfection and pathways projecting from MLR. Illumination through optical fiber excites MLR neuron below. (B) Locomotion velocity elicited by optogenetic stimulation (green bar) at $20 \mathrm{~Hz}$ (red) and failure of locomotion at $10 \mathrm{~Hz}$ (blue). (C) Increase in $\gamma$ band local field potential in V1 with 10-Hz MLR stimulation. (D) Example of increase in visual response with 10-Hz MLR stimulation, which did not elicit locomotion. (E) Group data showing average increase in visual response with 10-Hz MLR stimulation. (Data from Lee et al. 2014.)

ulus frequency was lowered to $10 \mathrm{~Hz}$, stimulation no longer evoked locomotion, but still drove the cortex into the high-gain state. This finding that MLR stimulation below the threshold for eliciting movement could mimic the effect of locomotion on the visual cortex in stationary animals suggests that change in the gain of visual cortical responses is produced by an ascending projection of the MLR to the forebrain, not by the descending projections of the MLR to motor regions that make the legs move. Consistent with this idea, optogenetic stimulation of in the basal forebrain of these same animals showed that activating the terminals of the MLR cells there could partially mimic the effect of locomotion on cortical gain (Lee et al. 2014).

\section{THE DISINHIBITORY CIRCUIT IN VISUAL CORTEX THAT ENHANCES RESPONSES DURING LOCOMOTION}

To locate the cortical portion of the circuit by which locomotion increases the responses of visual cortical neurons, we searched for neurons that responded to locomotion in the dark using a variety of mouse lines in which particular types of neurons were altered to express Cre recombinase (Fu et al. 2014). When these Cre lines were crossed with the Ai14 mouse line that conditionally expresses the fluorescent protein tdTomato, the selected cell type was made visible by its red fluorescence. Using the calcium indicator Oregon Green BAPTA-AM (OGB), we could use two-photon microscopy in alert animals to search the upper cortical layers for a cell type that responded to locomotion in the dark. A minor type of inhibitory neuron that expresses vasoactive intestinal peptide (VIP) had a strong, consistent, and sustained response to locomotion (Fig. 7). More than $97 \%$ of the nonVIP cells did not respond to locomotion in the dark. This finding suggested that the VIP cells, which were known to have nicotinic cholinergic receptors (Porter et al. 1999), were the starting point within the cortex for the regulation of cortical gain by locomotion.

This finding was initially puzzling, for why would activating an inhibitory cell cause the responses of excitatory cells to increase? Findings in vitro from other laboratories (Pfeffer et al. 2013), however, had shown the major output of VIP cells to be directed not to excitatory cells but to another class of inhibitory neurons, the somatostatin (SST) cells. Activating VIP cells would therefore be thought to reduce the activity of SST cells and thereby relieve the majority of excitatory cells from inhibition, allowing them to respond more strongly to visual stimuli. To test this idea, we made calcium recordings from SST cells in the dark. As predicted, most of them were suppressed by locomotion (Fig. 7).

Two experiments provided additional evidence that the VIP-SST disinhibitory circuit was responsible for putting the cortex into the high-gain state during locomotion (Fu et al. 2014). First, we transfected superficial layer VIP neurons with a form of channelrhodopsin. Optogenetic stimulation of the VIP neurons in stationary mice mimicked the effect of locomotion on responses of excitatory cells to visual stimuli. This finding showed that activating the VIP-SST circuit was sufficient to account for the effect of locomotion on cortical gain. To block the response of VIP cells to locomotion, we used a cruder technique: damaging labeled cells in VIP-Cre; Ai14 mice, in which the VIP cells are red. We first measured the effect of locomotion on the visual responses of a number of excitatory cells made visible using OGB in a single two-photon microscopic field and observed the normal increase in gain. We then focused the laser, one by one, on each VIP cell in the field, figuratively "blowing it away" and causing it to swell. After such photolytic damage to the VIP cells, we measured the visual responses of the same excitatory cells a second time during locomo- 


\section{M.P. STRYKER}

A

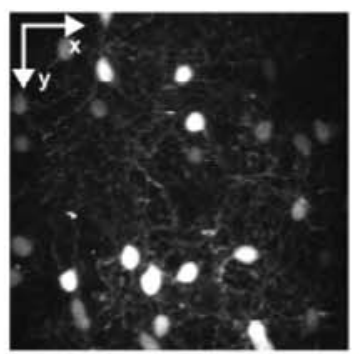

B

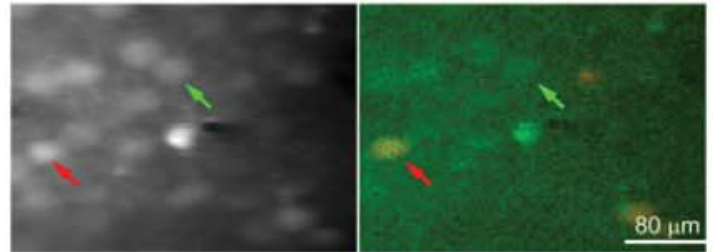

C
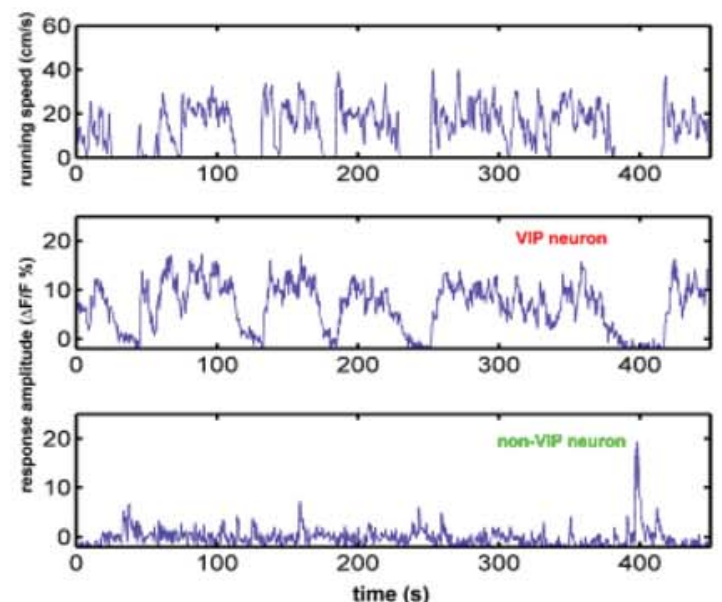

D
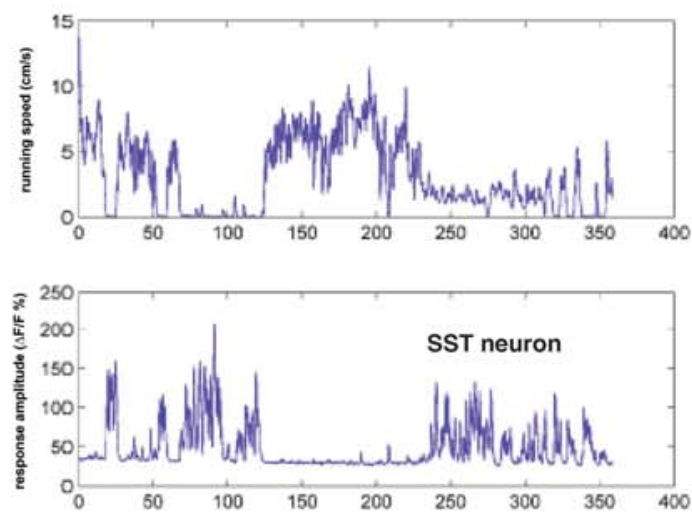

E

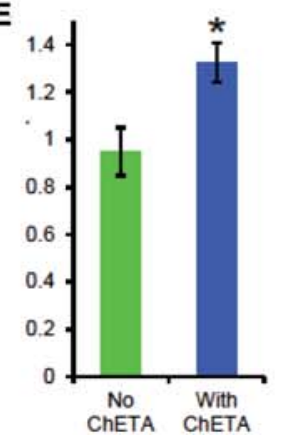

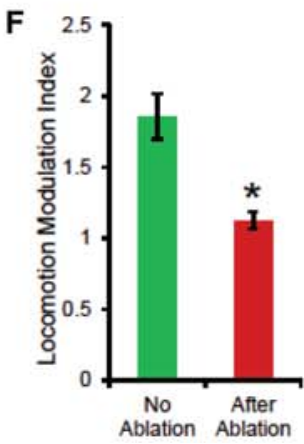

tion and while the mouse was stationary. The visual responses remained strong and selective, just as they were before the damage, but they were no longer enhanced by locomotion, so that the gain of the cortex was no longer increased. This finding shows that the VIP-SST circuit is necessary for the effect of locomotion on cortical gain.

To determine the source of the input conveying the locomotion signal to the VIP cells in visual cortex, we used a technique from the Callaway Laboratory that takes advantage of a modified rabies virus for tracing the monosynaptic inputs to a defined population of cells (Wickersham et al. 2007). We transfected the superficial layer VIP cells in a small region of visual cortex using the tracer virus and naively expected to see the retrograde label in a large population of cells in the motor cortex. Instead, very few cells in the motor cortex were labeled, and the most prominent projection was from the horizontal limb of the nucleus of the diagonal band of Broca (nDBB), a basal forebrain area containing cholinergic neurons that is within or near the region receiving projections from the MLR (not yet confirmed by double-tracer experiments). These cholinergic cells were likely candidates for conveying the locomotor input to the VIP cells. To test this notion further, we measured the effect of infusing nicotinic cholinergic blockers on the responses of VIP cells to locomotion and on the effect of locomotion on excitatory visual responses. Nicotinic blockade reduced both effects by more than two-thirds (Fu et al. 2014).

These findings are consistent with a circuit in which the locomotor signal from the MLR is conveyed via the $\mathrm{nDBB}$ to activate the nicotinic receptors of superficial VIP inhibitory cells of the primary visual cortex, which then inhibit the local SST cells and thereby disinhibit the excitatory cells of the visual cortex, making their visual responses larger but not altering their selectivity. This circuit can explain the influence of locomotion on cortical state and is consistent with many previous reports of cholinergic modulation of neural responses (Metherate and Weinberger 1989; Pinto et al. 2013). Because the nicotinic antagonists did not completely block the effect of locomotion in visual cortex, other neuromodulators may also play roles that have yet to be demonstrated in mouse visual cortex.

Figure 7. VIP cells carry the signal of locomotion to visual cortex to regulate cortical gain through inhibition of SST cells. $(A)$ VIP cells in surface view of layer 2 (left) and in coronal view (right) visualized using two-photon microscopy in vivo of VIPCre-Ai14 mice. $(B)$ VIP and non-VIP cells filled with Oregon Green BAPTA-AM to permit calcium recording, shown in green channel (left) and merge red and green channels (right). $(C)$ Calcium responses of a VIP- and a non-VIP-cell in relation to locomotion in the dark. $(D)$ Calcium responses of an SST cell expressing the indicator GCaMP6 in relation to locomotion in the dark. $(E)$ Optogenetic activation of VIP cells expressing a form of channelrhodopsin increases cortical gain (With ChETA, blue), whereas similar stimulation in VIP cells without channelrhodopsin (No ChETA, green) does not. $(F)$ Photodynamic damage to VIP cells (After ablation, red) blocks increase in cortical gain produced by locomotion before damage (No ablation, green). (Data from Fu et al. 2014.) 


\section{IS ENHANCED PLASTICITY THE PRODUCT OF AEROBIC EXERCISE OR THE HIGH-GAIN STATE OF VISUAL CORTEX?}

Is this VIP-SST disinhibitory circuit responsible for the dramatic effects of locomotion on adult plasticity described above (Kaneko and Stryker 2014)? Locomotion, in common with other forms of aerobic exercise, might conceivably affect cortical plasticity via multiple mechanisms, including those associated with increases in metabolism, such as blood flow and transport through astrocytes. Indeed, VIP cells themselves might participate in signaling relevant to metabolic activity because they release not only the inhibitory neurotransmitter GABA, but also the peptide VIP. The most powerful effect of VIP on the brain appears to be on glycogenolysis in astrocytes, producing lactate that acts as an energy source (Magistretti et al. 1981). To determine whether locomotion enhances visual cortical plasticity via its aerobic exercise effects or via the increased visual responses produced by the VIP-SST disinhibitory circuit, we sought to block this circuit in running mice and to activate it in stationary mice and measure the resulting effects on cortical plasticity (Fu et al. 2015). We blocked this circuit in running mice using a virus that conditionally expressed tetanus toxin (a generous gift from the Sudhof Laboratory) in VIP-Cre mice. Tetanus toxin blocks the release of neurotransmitter in the cells that express it; it did not alter the survival of the VIP cells during the course of these experiments. Experimental and control mice were subjected to exactly the same regimen of more than 4 mo of monocular visual deprivation beginning in the critical period, as described above (Kaneko and Stryker 2014). After opening the deprived eye, the mice were allowed to run on the trackball while viewing high-contrast visual stimuli. As previously, the control mice rapidly recovered normal levels of visual responsiveness to the stimuli to which they were exposed. In contrast, the VIPtetanus toxin mice did not recover responses any more than the mice kept in their home cages. This finding indicates that the release of neurotransmitter specifically from VIP cells is essential for the enhancement of adult plasticity by running in mouse visual cortex (Fig. 8) (Fu et al. 2015).

We also explored whether locomotion could enhance plasticity in a short-term assay. In normal adult mice, occluding the vision of the contralateral for $4-5 \mathrm{~d}$ has no effect on visual responses to stimulation of either eye; however, monocular deprivation continued past $7 \mathrm{~d}$ causes visual cortical responses to stimulation through the open, nondeprived eye to increase while responses through the deprived eye remain the same. This form of adult plasticity is distinct from the plasticity observed during the critical period, where the earliest and most dramatic change is the reduction in responses to deprived-eye stimulation. In normal adult animals that were monocularly deprived and then viewed high-contrast visual stimuli through the ipsilateral eye during locomotion, an increase in response to the open eye was evident after 4-5 d. Tetanus toxin expression in VIP cells prevented this increase. When we did the complementary experiment of optogenetically activating VIP cells in stationary adult mice during short-term monocular deprivation, an increase in open-eye response similar to that in running mice was observed, demonstrating that VIP cell activation without aerobic exercise could mimic the effects of locomotion. Finally, the VIP-SST disinhibitory circuit is hypothesized to alter plasticity via the suppression of SST cell activity by VIP cells during locomotion. To test this hypothesis, we expressed tetanus toxin in SST cells in adult mice and then performed shortterm monocular deprivation while the mice were stationary. Blocking SST-cell output by this treatment, like optogenetically activating VIP cells, produced the adult form of plasticity, mimicking the effect of locomotion, but without aerobic activation. Taken together, these findings indicate that the effect of running on adult plasticity is principally mediated through the VIP-SST disinhibitory circuit rather than by some other effect of aerobic activity (Fu et al. 2015).
A

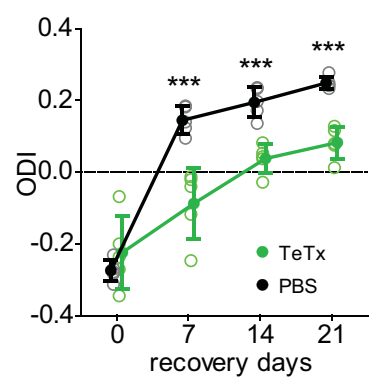

B

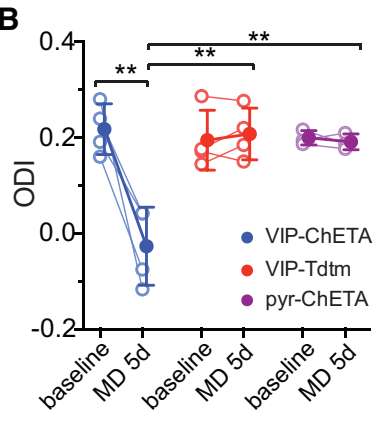

C

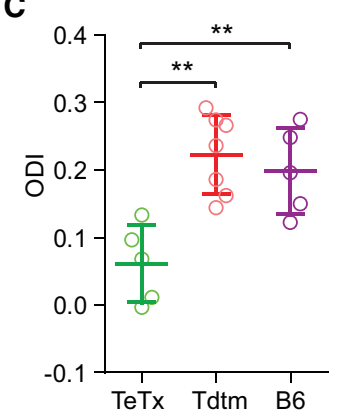

Figure 8. Blockade and activation of VIP-SST disinhibitory circuit regulate cortical plasticity. (A) Expression of tetanus toxin to block synaptic release from VIP cells (green) prevents the enhancement of recovery from long-term monocular deprivation by locomotion (black). Compare with Figure 5. (B) Optogenetic activation of VIP cells (VIP-ChETA, blue) during $5 \mathrm{~d}$ of monocular deprivation in stationary adult mice produces plasticity; similar stimulation of excitatory neurons (pyr-ChETA, magenta) or in VIP cells expressing tdTomato without channelrhodopsin (VIP-Tdtm, red) does not. (C) Expression of tetanus toxin in SST cells (TeTx, green) during $5 \mathrm{~d}$ of monocular deprivation in stationary adult mice produces plasticity; expression of tdTomato (Tdtm, red) does not, yielding results similar to 5-d MD in C57BL6/J control mice (B6, magenta). (ODI) Ocular dominance index of relative response to the two eyes. (Data from Fu et al. 2015.) 
The fact that VIP cells rather than some other type of inhibitory neurons increase visual cortical gain and enhance adult plasticity in a manner similar to the aerobic activity of locomotion may be a coincidence, or it may suggest that the action of VIP peptide in mobilizing lactate metabolism through glycogenolysis is a normal part of the effect of aerobic exercise on the brain. It is plausible that evolution has adapted this form of signaling to increase the metabolic capacity of the brain during the high-gain state, even when sedentary, or perhaps VIP peptide operates as a rapid signal that anticipates other consequences of exercise. It will be interesting to see whether the VIP peptide alone, in the absence of VIPSST disinhibition of excitatory pathways, might affect cortical plasticity, as experiments in the hippocampus suggest (Donato et al. 2013). Experiments to illuminate this issue in the visual cortex are in progress.

\section{CONCLUSION}

Anatomical elements of the VIP-SST disinhibitory circuit exist in many cortical areas and in many mammalian species including humans. At the present time, we do not know how this circuit operates in other cortical areas and species or whether it enhances neural activity or plasticity. Evidence for cholinergic modulation of plasticity is widespread, although some pharmacological studies implicate muscarinic rather than the nicotinic actions that we see in the VIP cells of the superficial layers of the mouse visual cortex.

Neuromodulators other than acetylcholine are also thought to influence neural plasticity. There is longstanding evidence for effects of norepinephrine and serotonin on plasticity in the visual cortex (Bear and Singer 1986; Imamura and Kasamatsu 1989; Kojic et al. 2001) and evidence for actions of dopamine on plasticity in many brain areas (Jay 2003). Indeed, early studies involving stimulation or lesions to the midbrain reticular formation, in areas near or overlapping with the MLR, were thought to implicate actions of these other neuromodulatory systems on plasticity (Singer and Rauschecker 1982). Whether or how these other neuromodulatory systems interact with the VIP-SST disinhibitory circuit is not known. There may well be multiple sites in the cortical circuit that are modulated to alter plasticity.

Deep brain electrical stimulation in the human homolog of the MLR at frequencies similar to those we used here in mice has been explored as therapy for movement disorders such as Parkinson's disease (Stefani et al. 2007). When stimulated, patients commonly report a heightened feeling of alertness (Stefani et al. 2013). It is plausible that alertness would be the subjective feeling associated with the high-gate state of cortex; however, we do not know whether locomotion induces a high-gain state in human visual cortex. In the mouse, a dramatic and sustained increase in the magnitude of $\gamma$ oscillations near $60 \mathrm{~Hz}$ accompanies the high-gain state produced by locomotion or MLR stimulation. Experiments are under way to measure whether $\gamma$ power in human visual cortex is increased by locomotion. Those, in addition to psychophysical experiments to determine whether perception is enhanced by locomotion, should resolve whether the VIP-SST disinhibitory circuit is activated in humans as it is in the mouse.

Considering the operation of the visual cortex from an information processing point of view, modulation of cortical gain by the VIP-SST disinhibitiory circuit has the tremendously desirable feature that it separates the channels that convey the stimulus specificity of cortical neurons from those that merely modulate their gain. The visually driven inputs are unchanged by locomotion, except insofar as the visual field is moved across the retinae. The filter properties of the cortical network may remain intact, but the responses of neurons become greater because of their relief from nonvisual inhibition. Other circuits in the visual cortex appear to operate similarly by disinhibition. For example, the modulation of cortical gain by activity in layer 6 , thought to be responsible for some of the spatial properties of cortical neurons, has recently been shown to be achieved through the action of a special class of parvalbumin-containing inhibitory neurons with somata in layer 6 and axons that terminate in the other cortical layers (Olsen et al. 2012; Bortone et al. 2014).

The notion of the ascending reticular activating system that emerged from studies of anesthetized animals was a useful concept for many years, bringing together phenomena of neuromodulation, rhythmic activity, plasticity, movement, and alertness (Moruzzi and Magoun 1949; Skinner et al. 2004). We are now in a position, in mice at least, to dissect the cell types and circuitry responsible for this wide range of phenomena that come together in a small area of the midbrain and have such wide effects. It is not yet clear which of these phenomena are necessarily associated by common circuitry and which by the happenstance of proximity or common pharmacology that leads to apparent association by common effects of electrical stimulation, lesions, or drugs. One can be optimistic that the tools needed to decide these issues are in place or will be soon.

\section{ACKNOWLEDGMENTS}

I gratefully acknowledge the people who have performed most of the research described here, especially Megumi Kaneko, Yu Fu, Cristopher Niell, Moses Lee, and Sebastian Espinosa, and the support, principally from the National Eye Institute under R01EY02874 and from the Simons Collaboration on the Global Brain, that made it possible.

\section{REFERENCES}

Antonini A, Stryker MP. 1993. Rapid remodeling of axonal arbors in the visual cortex. Science 260: 1819-1821.

Antonini A, Fagiolini M, Stryker MP. 1999. Anatomical correlates of functional plasticity in mouse visual cortex. J. Neurosci 19: 4388-4406. 
Bear MF, Singer W. 1986. Modulation of visual cortical plasticity by acetylcholine and noradrenaline. Nature 320: 172 176.

Bortone DS, Olsen SR, Scanziani M. 2014. Translaminar inhibitory cells recruited by layer 6 corticothalamic neurons suppress visual cortex. Neuron 82: 474-485.

Dombeck DA, Khabbaz AN, Collman F, Adelman TL, Tank DW. 2007. Imaging large-scale neural activity with cellular resolution in awake, mobile mice. Neuron 56: 43-57.

Donato F, Rompani SB, Caroni P. 2013. Parvalbumin-expressing basket-cell network plasticity induced by experience regulates adult learning. Nature 504: 272-276.

Fu Y, Tucciarone JM, Espinosa JS, Sheng N, Darcy DP, Nicoll RA, Huang ZJ, Stryker MP. 2014. A cortical circuit for gain control by behavioral state. Cell 156: 1139-1152.

Fu Y, Kaneko M, Tang Y, Alvarez-Buylla A, Stryker MP. 2015. A cortical disinhibitory circuit for enhancing adult plasticity. Elife 4: e05558.

Garcia-Rill E. 1991. The pedunculopontine nucleus. Prog Neurobiol 36: 363-389.

Gordon JA, Stryker MP. 1996. Experience-dependent plasticity of binocular responses in the primary visual cortex of the mouse. J Neurosci 16: 3274-3286.

Greifzu F, Pielecka-Fortuna J, Kalogeraki E, Krempler K, Favaro PD, Schluter OM, Lowel S. 2014. Environmental enrichment extends ocular dominance plasticity into adulthood and protects from stroke-induced impairments of plasticity. Proc Natl Acad Sci 111: 1150-1155.

Hubel DH, Wiesel TN. 1962. Receptive fields, binocular interaction and functional architecture in the cat's visual cortex. $J$ Physiol 160: 106-154.

Imamura K, Kasamatsu T. 1989. Interaction of noradrenergic and cholinergic systems in regulation of ocular dominance plasticity. Neurosci Res 6: 519-536.

Jay TM. 2003. Dopamine: A potential substrate for synaptic plasticity and memory mechanisms. Prog Neurobiol 69: 375-390.

Kalatsky VA, Stryker MP. 2003. New paradigm for optical imaging: Temporally encoded maps of intrinsic signal. Neuron 38: 529-545.

Kaneko M, Stryker MP. 2014. Sensory experience during locomotion promotes recovery of function in adult visual cortex. Elife 3: 02798 .

Kaneko M, Hanover JL, England PM, Stryker MP. 2008. TrkB kinase required for recovery but not loss of cortical responses following monocular deprivation. Nat Neurosci 11: 497-504.

Kojic L, Gu Q, Douglas RM, Cynader MS. 2001. Laminar distribution of cholinergic- and serotonergic-dependent plasticity within kitten visual cortex. Brain Res Dev Brain Res 126: $157-162$.

Lee AM, Hoy JL, Bonci A, Wilbrecht L, Stryker MP, Niell CM. 2014. Identification of a brainstem circuit regulating visual cortical state in parallel with locomotion. Neuron 83: 455-466.

Magistretti PJ, Morrison JH, Shoemaker WJ, Sapin V, Bloom FE. 1981. Vasoactive intestinal polypeptide induces glycogenolysis in mouse cortical slices: A possible regulatory mechanism for the local control of energy metabolism. Proc Natl Acad Sci 78: 6535-6539.

Maya Vetencourt JF, Sale A, Viegi A, Baroncelli L, De Pasquale R, O’Leary OF, Castren E, Maffei L. 2008. The antidepressant fluoxetine restores plasticity in the adult visual cortex. Science 320: 385-388.

Metherate R, Weinberger NM. 1989. Acetylcholine produces stimulus-specific receptive field alterations in cat auditory cortex. Brain Res 480: 372-377.

Moruzzi G, Magoun HW. 1949. Brain stem reticular formation and activation of the EEG. Electroencephalogr Clin Neurophysiol 1: 455-473.

Nauta WJH, Kuypers H. 1958. Some ascending pathways in the brain stem reticular formation. In Reticular formation of the brain (ed. Jasper HH, Proctor LD, Knighton RS, et al.), pp. 3-30. Little, Brown, Boston.

Niell CM, Stryker MP. 2008. Highly selective receptive fields in mouse visual cortex. J Neurosci 28: 7520-7536.

Niell CM, Stryker MP. 2010. Modulation of visual responses by behavioral state in mouse visual cortex. Neuron 65: 472-479.

Olsen SR, Bortone DS, Adesnik H, Scanziani M. 2012. Gain control by layer six in cortical circuits of vision. Nature 483: 47-52.

Orlovskii GN, Severin FV, Shik ML. 1966. Locomotion induced by stimulation of the mesencephalon. Dokl Akad Nauk SSSR 169: $1223-1226$.

Pfeffer CK, Xue M, He M, Huang ZJ, Scanziani M. 2013. Inhibition of inhibition in visual cortex: The logic of connections between molecularly distinct interneurons. Nat Neurosci 16: 1068-1076.

Pinto L, Goard MJ, Estandian D, Xu M, Kwan AC, Lee SH, Harrison TC, Feng G, Dan Y. 2013. Fast modulation of visual perception by basal forebrain cholinergic neurons. Nat Neurosci 16: $1857-1863$.

Porter JT, Cauli B, Tsuzuki K, Lambolez B, Rossier J, Audinat E. 1999. Selective excitation of subtypes of neocortical interneurons by nicotinic receptors. J Neurosci 19: 5228-5235.

Sale A, Maya Vetencourt JF, Medini P, Cenni MC, Baroncelli L, De Pasquale R, Maffei L. 2007. Environmental enrichment in adulthood promotes amblyopia recovery through a reduction of intracortical inhibition. Nat Neurosci 10: 679-681.

Sato M, Stryker MP. 2008. Distinctive features of adult ocular dominance plasticity. J Neurosci 28: 10278-10286.

Singer W, Rauschecker JP. 1982. Central core control of developmental plasticity in the kitten visual cortex: II. Electrical activation of mesencephalic and diencephalic projections. Exp Brain Res 47: 223-233.

Skinner RD, Homma Y, Garcia-Rill E. 2004. Arousal mechanisms related to posture and locomotion: 2. Ascending modulation. Prog Brain Res 143: 291-298.

Stefani A, Lozano AM, Peppe A, Stanzione P, Galati S, Tropepi D, Pierantozzi M, Brusa L, Scarnati E, Mazzone P. 2007. Bilateral deep brain stimulation of the pedunculopontine and subthalamic nuclei in severe Parkinson's disease. Brain 130: $1596-1607$.

Stefani A, Peppe A, Galati S, Bassi MS, D'Angelo V, Pierantozzi M. 2013. The serendipity case of the pedunculopontine nucleus low-frequency brain stimulation: Chasing a gait response, finding sleep, and cognition improvement. Front Neurol 4: 68.

Wickersham IR, Lyon DC, Barnard RJ, Mori T, Finke S, Conzelmann KK, Young JA, Callaway EM. 2007. Monosynaptic restriction of transsynaptic tracing from single, genetically targeted neurons. Neuron 53: 639-647. 


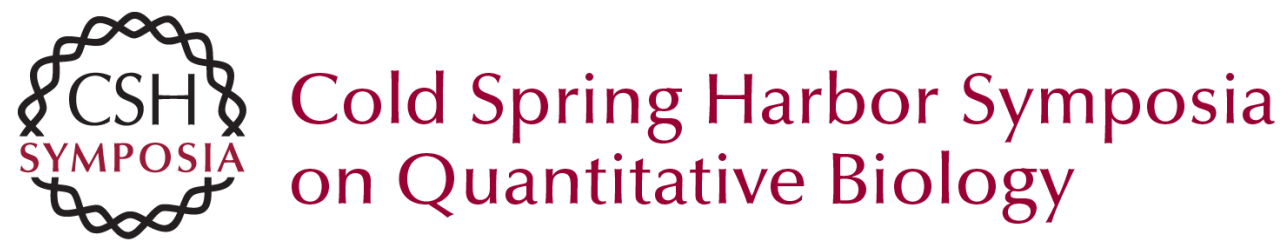

\section{A Neural Circuit That Controls Cortical State, Plasticity, and the Gain of Sensory Responses in Mouse}

Michael P. Stryker

Cold Spring Harb Symp Quant Biol 2014 79: 1-9 originally published online May 6, 2015 Access the most recent version at doi:10.1101/sqb.2014.79.024927

References This article cites 37 articles, 9 of which can be accessed free at: http://symposium.cshlp.org/content/79/1.full.html\#ref-list-1

License

Email Alerting Receive free email alerts when new articles cite this article - sign up in Service the box at the top right corner of the article or click here. 